\title{
La contribución de Richard Rorty para una pedagogía democrática en contexto de posmodernidad
}

\author{
Richard Rorty's contribution to a democratic \\ pedagogy in a postmodern context
}

Juan José Ramírez ${ }^{1}$

\begin{abstract}
Resumen: ¿Son excluyentes los rasgos filosóficos de la posmodernidad y las exigencias propias de un discurso pedagógico? ¿Puede edificarse una pedagogía posmoderna? El presente trabajo intenta esbozar respuestas a estos interrogantes. Se cree que el escenario cultural posmoderno no constituye, necesariamente, un nicho para el anuncio de la muerte de la pedagogía. Algunos debates posmodernos en torno a la educación pueden contribuir a un replanteo de la disciplina desde una geografía portadora de nuevas significaciones.

Desde el ámbito de la filosofía posmoderna, se ensayan algunos recorridos que albergan la posibilidad de nuevas contribuciones a la pedagogía. La idea rortyana de una "educación sentimental" es portadora de herramientas didácticas para nuevas contingencias en el quehacer educativo y la reflexión pedagógica.

El tipo de "educación sentimental" impulsado por el neopragmatista tiene su rasgo distintivo en una propuesta pedagógica de carácter ironista que no pretende ser asimilada por marco epistemológico alguno. Se trata de una propuesta educativa que, antes que fundarse en una naturaleza antropológica, se articula con una disposición edificante de corte hermenéutico.
\end{abstract}

Palabras clave: hermenéutica, democracia, sensibilidad, educación.

\footnotetext{
${ }^{1}$ Doctor en Ciencia Política. Docente de la Universidad Católica de Córdoba, Argentina. Correo electrónico: jjrcba@hotmail.com.

Diálogos Pedagágicas. ISSN en línea: 2524-9274.

Año XVIII, No 35, abril - septiembre 2020. Pág. 24-36.

DOI: http://dx.doi.org/10.22529/dp.2020.18(35)02 / Recibido: 1-10-2019 / Aprobado: 9-03-2020.
}

(c) (1) $\odot$ Artículo publicado bajo Licencia Creative Commons Atribución-NoComercial-SinDerivar. (C) Universidad Católica de Córdoba. 


\begin{abstract}
Are the philosophical features of postmodernism and the demands of a pedagogical discourse exclusive? Can a postmodern pedagogy be built? The present work tries to outline answers to these questions. It is believed that the postmodern cultural scenario does not necessarily constitute a niche for the announcement of the death of pedagogy. Some postmodern debates about education can contribute to rethink the discipline from a geography including new meanings.

From the field of postmodern philosophy, some routes that host the possibility of new contributions to pedagogy are rehearsed. The idea of Rorty of a "sentimental education" carries teaching tools for new contingencies in educational work and pedagogical reflection.

The type of "sentimental education" promoted by the neopragmatist has its distinctive feature in an educational proposal of an ironist character that is not intended to be assimilated by any epistemological framework. It is an educational proposal that, rather than being based on an anthropological nature, articulates itself with an edifying disposition of a hermeneutical nature.
\end{abstract}

Keywords: hermeneutics, democracy, sensibility, education.

\title{
1. Pedagogía y posmodernidad
}

En el marco de la discusión respecto de la posibilidad de una pedagogía de corte posmoderno, Ayuste González y Trilla Bernet (2005) consideran imposible la afirmación de dicha pedagogía. Ellos entienden que los rasgos propios de la cultura posmoderna excluyen las exigencias que le son atribuidas al tipo de reflexión que pretende constituir una pedagogía.

Ayuste González y Trilla Bernet señalan que aquella reflexión que tenga la pretensión de configurar una pedagogía debe satisfacer, al menos, tres requisitos: a) debe ofrecerse como una reflexión sobre la educación en general y no solo sobre algún tópico específico de las problemáticas educativas, b) debe comportar la voluntad de una coherencia interna discursiva y c) no debe tener un carácter meramente descriptivo, sino que, además, debe incluir contenidos normativos.

Los primeros dos requisitos que se le exigen a un discurso para ser pedagógico constituyen exigencias propias de cualquier ciencia moderna. La generalidad y la coherencia son, entre otras, exigencias epistemológicas propias de una disciplina científica. En lo que hace al tercer requisito (la necesidad de comportar un carácter normativo y no solo descriptivo), este acerca la idea de pedagogía de los investigadores, no a una disciplina de carácter fáctico y, entonces, empírico, sino a un saber teñido de rasgos emparentados con la denominada filosofía práctica. Si la pedagogía es considerada como un saber que no debe autolimitarse a sus posibilidades descriptivas respecto del fenómeno educativo, sino que, además debe poder señalar el "deber ser" de ese fenómeno, entonces, estamos en presencia de una exigencia disciplinaria de carácter ético-político. Esta exigencia renueva las discusiones en torno a qué tipo de saber comporta la pedagogía. 
Para Ayuste González y Trilla Bernet (2005), "no hay pedagogías relevantes elaboradas desde el pensamiento postmoderno [...] y en rigor no puede haberlas" (p. 231). Ellos creen que la idea de pedagogía se encuentra indisolublemente unida a los valores de la Ilustración. Sin ideas como las de progreso, racionalidad o conocimiento, parece no ser posible pensar en términos pedagógicos. En este punto, los investigadores parecen liar la idea de pedagogía con principios, requisitos y un aparato conceptual argumentativo particular; para ellos, no hay pedagogía al margen de un léxico que comporte esas exigencias puntuales.

La historia de la pedagogía revela que la reflexión en torno a la educación excede el marco histórico-cultural moderno. En este sentido, parece conveniente justificar las razones por las que se considera que este saber debe estar enlazado, de manera necesaria, con las categorías propias de un momento histórico particular de la cultura.

Las reflexiones posmodernas en torno al fenómeno educativo son, para Ayuste González y Trilla Bernet, meros relatos discursivos sobre la educación; aquellas reflexiones no alcanzan el rango epistemológico exigido para constituirse en pedagogías. Los discursos educativos posmodernos son meramente descriptivos, o bien se orientan a esbozar denuncias y críticas respecto de alguna arista del hecho educativo. Según Ayuste González y Trilla Bernet, los discursos educativos de la posmodernidad no avanzan más allá de lo que lo hiciera alguna pedagogía moderna; y si acaso algún discurso tuviera esa pretensión, su intención parece condenada a sucumbir en contradicciones con los presupuestos propios de la filosofía posmoderna. La idea de una pedagogía posmoderna parece ser, en este contexto, una contradicción en sí misma. La conclusión de los profesores de BarceIona posee un rigor lógico impecable; sin embargo, las premisas de las que emana aquella conclusión no precisan, de modo inequívoco, la noción de posmodernidad que comportan. Los catalanes no señalan explícitamente si refieren a la condición posmoderna del saber descripta por Jean Francois Lyotard, al neopragmatismo postanalítico de Richard Rorty, a la hermenéutica nihilista de Gianni Vattimo o a una producción cultural que posea cierto aire de familia con alguno de los denominados pensadores posmodernos.

El carácter antifundacionalista, el abandono de la universalidad y la consiguiente autolimitación para esbozar horizontes teleológicos, que los pedagogos atribuyen al pensamiento posmoderno, parecen ser características de un tipo de racionalidad que, según Ayuste González y Trilla Bernet, están reñidas con la posibilidad de una auténtica reflexión pedagógica.

Xavier Laudo Castillo (2011), en contraposición con la perspectiva de Ayuste González y Trilla Bernet, abona la posibilidad de una pedagogía con rasgos posmodernos. Para él, no hay, en los rasgos generales y comunes del pensamiento posmoderno, herramientas conceptuales que obturen la posibilidad de una pedagogía posmoderna. Castillo no solo descree de aquellos obstáculos epistemológicos, sino que, además, echa luz sobre los rasgos epocales de una cultura posmoderna y muestra que, en cualquier caso, no están reñidos con las posibilidades pedagógicas, sino que contribuirían a una nueva forma de entender la disciplina en cuestión. En este contexto, la pedagogía no exige necesariamente 
una racionalidad entendida como moderna ${ }^{2}$, sino que está abierta a nuevas formas de entender la racionalidad que impulsa u orienta el saber pedagógico.

Para Castillo, parece quedar claro que la intención de Ayuste González y Trilla Bernet no ha sido otra que atribuir al pensamiento posmoderno un tipo de meta narrativa que clausura las posibilidades de una disciplina que se ha erigido, históricamente, sobre una metanarrativa olvidada y en desuso. El valioso aporte de Castillo a la discusión radica en el desmontaje de todos y cada uno de los obstáculos que Ayuste González y Trilla Bernet han sugerido como impedimentos objetivos para la posibilidad de una pedagogía posmoderna.

El crítico norteamericano Henry Giroux es señalado por Ayuste González y Trilla Bernet (2005) como uno de los teóricos de la educación que representa las ideas de la izquierda liberal progresista de Norteamérica. La obra de Giroux constituye, para ellos, lo más próximo que puede encontrarse a una pedagogía posmoderna. Al mismo tiempo, Ayuste González y Trilla Bernet se encargan de señalar, con justa razón, que esa proximidad de ningún modo permite identificar el pensamiento crítico de Giroux con los rasgos propios del pensamiento posmoderno.

La reflexión crítica de Giroux concibe la educación como una práctica cultural, política y social. En esa línea, se realiza un cuestionamiento a la escolaridad como forma de subordinación que promueve inequidades sociales. El hecho de que Giroux se permita entablar conversaciones con el pensamiento posmoderno no lo convierte en un pensador posmoderno. La pedagogía crítica de Giroux pretende afirmarse como un espacio de intersección entre modernidad y posmodernidad que rescata lo mejor de ambas geografías culturales. La pedagogía crítica del estadounidense constituye una renovada crítica cultural de la modernidad. Para esa renovación, aquella crítica se nutre de herramientas que son propias de la cultura posmoderna; sin embargo, la crítica misma sigue siendo moderna. Se trata de una crítica que sigue impulsando los valores del mundo moderno y que se revela a sí misma en franca oposición con algunos de los principios del pensamiento de la posmodernidad. El sentido de la crítica moderna, en modo alguno, puede ser equivalente a las formas críticas de la posmodernidad. Cualquier forma de crítica en contextos de modernidad comporta la proposición de una alternativa positiva y superadora respecto de lo que se ha criticado. La crítica, en clave posmoderna, no puede albergar aquellas pretensiones sin portar una contradicción. La crítica posmoderna se identifica con un desmontaje de lo que se critica y la sugerencia de olvido respecto de lo criticado.

\footnotetext{
2 Rorty distingue dos sentidos del término "racionalidad". El primero de esos sentidos es el que refleja lo que, en este trabajo, se entiende por "racionalidad moderna". En un sentido, "ser racional es ser metódico, es decir tener criterios de éxito fijados de antemano" (Rorty, 1996, p. 59). Es plausible considerar a la ciencia natural como el paradigma de la racionalidad, si se considera al científico como alguien que está dispuesto a abandonar cualquier hipótesis, a causa del resultado desfavorable de un único experimento en relación con ella. Desde esta óptica, la actividad del científico no comporta mérito alguno más que su disposición para ajustarse al uso de determinadas reglas. En otro sentido racional significa algo como sensato o razonable. En este caso, el término "designa un conjunto de virtudes morales: tolerancia, respeto a las opiniones de quienes nos rodean, disposición a escuchar y recurso a la persuasión antes que a la fuerza" (Rorty, 1996, p. 59).
} 


\section{Un debate pedagógico entre Henry Giroux y Richard Rorty}

Los rasgos que evidencian el carácter moderno del pensamiento de Giroux se revelan con claridad en la disputa que este sostiene con algunos sectores de la filosofía posmoderna norteamericana. Para Giroux:

La filosofía posmoderna, y en especial la variante liberal norteamericana ha tendido a sacrificar el desarrollo de un discurso pedagógico de la ética, el albedrío y la política, en aras de un lenguaje de la crítica orientado a la demolición de toda pretensión del conocimiento en cuanto certidumbre y primeros principios. (Giroux, 1993, p. 105)

Giroux entiende que el trabajo de Richard Rorty constituye una representación de la versión del antifundacionalismo norteamericano propio de una filosofía posmoderna y liberal. La perspectiva posmoderna de Rorty comporta, para Giroux, un lenguaje crítico que "menosprecia cualquier llamado a la esperanza" (Giroux, 1993, p. 106). Giroux considera que, en el pensamiento de Rorty, el conocimiento, y entonces también la sociedad, son considerados como un asunto meramente práctico que ha sido despojado de su carácter político y de las relaciones de poder que explicarían aquellas instituciones.

En la perspectiva del crítico cultural norteamericano, el filósofo posmoderno es portador de una ideología que amarra intencionalmente las posibilidades del intelectual en la vida de la comunidad. Para Rorty, la idea de una crítica ideológica es sinónimo de una intelectualidad estéril. Una auténtica crítica de las instituciones sociales solo se constituye en tal en la medida en que puede imaginar y proponer instituciones alternativas a aquellas de las que disponemos.

Para Giroux, Rorty entiende al intelectual como un sujeto despolitizado, alguien sin posibilidades de compromiso o de lucha social. La versión posmoderna que encarna el pragmatismo rortyano "es fundamentalmente antiutópico en el sentido de que socava y despolitiza el proyecto deweyano de vincular la voluntad pública con la idea de la democracia radical" (Giroux, 1993, p. 108).

Para Giroux, el antifundacionalismo rortyano puede constituir una herramienta útil para criticar las tradiciones y los códigos intelectuales que se legitiman mediante formas diversas de transcendentalismos; sin embargo, aquella disposición antifundacionalista obtura la posibilidad de ofrecer algún fundamento ético o político para denunciar el sufrimiento y las contradicciones de la sociedad moderna. Desde esta perspectiva, el intelectual rortyano es incapaz de portar herramientas morales o políticas para luchar por una sociedad más justa.

En clara alusión crítica a la posición posmoderna de Rorty, Giroux afirma:

Si se quiere que los conceptos de discurso moral y visión política se conviertan en algo más que otra ramificación flotante dentro de la comunidad de las conversaciones sociales, los educadores críticos tendrán que desplazarse más allá de las limitaciones teóricas e ideológicas que subyacen a los diversos enfoques derechistas y liberales frente a las cuestiones afines de la educación escolar, la moralidad y la autoridad. (Giroux, 1993, p. 110) 
Richard Rorty se ha manifestado, en numerosas ocasiones, como un pensador norteamericano liberal y antifundacionalista; sus ideas sintonizan claramente con los rasgos de lo que se ha dado en llamar filosofía posmoderna. No obstante lo anterior, el filósofo norteamericano nunca se ha considerado a sí mismo en términos de un pensador "derechista", y su perspectiva filosófica y política difícilmente pueda ser considerada como conservadora ${ }^{3}$.

La posición política de Rorty ha sido criticada tanto por pensadores de derecha como por intelectuales de izquierda (Rorty, 1998a); la crítica de Giroux se orienta en la dirección de estos últimos. Las diferencias de Rorty con la izquierda cultural norteamericana radican en las exigencias epistemológicas y lingüísticas que los cambios sociales requieren. Rorty cree que, para realizar la esperanza de una sociedad mejor, los intelectuales deben dejar de asumir que su función política es la de llevar a cabo una crítica radical de las instituciones, "a través de una sobrefilosofización del debate político" (Rorty, 1998b, p. 137).

El debate entre Giroux y Rorty bien podría centrarse en la forma y el alcance que debe adquirir la crítica social. Para Giroux, esta crítica solo es posible si adquiere la forma de una crítica radical. Para Rorty, en cambio, la crítica solo puede ser fructífera en la medida en que sea local y reformista. Para Giroux, la idea de una crítica reformista es insuficiente y estéril; para Rorty, la idea de una crítica radical redunda en aspiraciones epistemológicas totalitarias.

El pragmatista entiende que "el vocabulario de la política social democrática [...] no necesita de una mayor sofisticación por parte de los filósofos" (Rorty, 1993, p. 46) y podría decirse que lo mismo cuenta para cualquier sector de la cultura que quiera enriquecer las instituciones de la vida pública. Las contribuciones más eficaces para la justicia social pueden ser realizadas al interior del sistema democrático constitucional y estos aportes no requieren de un vocabulario filosófico excesivamente complejo o de cualquier otro vocabulario extrapolítico. Para una educación democrática tampoco se necesita de una pedagogía que apele a un léxico sofisticado.

\section{Educación y democracia en contextos de posmodernidad}

La educación es una de las instituciones que habitan el interior del sistema democrático. Cualquier intelectual preocupado por el costado público de las instituciones democráticas ha hecho referencias explícitas referidas a la educación. Richard Rorty, quizás impulsado por la obra de su pensador favorito, no constituye una excepción. El norteamericano, en tanto admirador de John Dewey y filósofo de la democracia, ha promovido la idea de una educación sentimental. Seguramente,

\footnotetext{
3 El pensamiento rortyano nunca vislumbró la necesidad de un marco institucional y político diferente del de Estados Unidos y esto se debe a que el propósito de su reflexión nunca fue escapar del patriotismo y las contingencias locales. La reflexión neopragmatista de Rorty está signada por un carácter etnocéntrico y un marcado rechazo de la idea de una racionalidad universal. Para un estudio pormenorizado, véase Rorty, 2005. Para justificar los elementos que hacen a la consideración de Rorty como un pensador de izquierda no conservador, véase además Rorty, 1998a.
} 
el asunto de si su propuesta educativa comporta o no una pedagogía es una disquisición que para el filósofo carece de importancia.

Los límites de las disciplinas que cohabitan una cultura son siempre difusos ${ }^{4}$ y no reflejan más que el carácter de los acuerdos que las diversas comunidades científicas han podido establecer. La demarcación de los límites de una esfera cultural es siempre autorreferencial para los miembros de aquellas comunidades; en este sentido, la distinción entre pedagogías y discursos educativos no encubre otra cosa que el interés por separar las propias y legítimas producciones pedagógicas de extrañas pseudopedagogías. Si nos despojamos del propósito de conmensurar las propuestas educativas según nuestros propios marcos referenciales, se podrá considerar a cualquier discurso vinculado a la educación como un intento válido por mejorar nuestras instituciones educativas y por hacer de la educación una herramienta más entre otros utensilios al servicio de la propia comunidad $^{5}$. La valoración de otros discursos educativos por su grado de acercamiento con el marco epistemológico de la propia pedagogía, en vez de enriquecer las posibilidades para articular discursos, estrecha nuestros marcos de referencia.

Los rasgos de una educación democrática se pueden identificar con los de una propuesta pedagógica que valore la aceptación de las diferencias y promueva, en los ciudadanos, conductas no violentas para con aquellos que no comparten algunas o muchas de las creencias propias. Para Rorty, y desde lo que se puede entender como una perspectiva posmoderna, el tipo de conducta que consideramos reprochable en una comunidad democrática no es una conducta irracional. El par racional-irracional no ha resultado un instrumento particularmente beneficioso para estimular la convivencia democrática entre ciudadanos con diferentes obsesiones privadas. Las creencias de aquellas personas cuyas conductas pueden parecer reprochables no son más o menos racionales que nuestras propias creencias. En el contexto de la posfilosofía rortyana, aquello que se considera racional está atado a las condiciones de justificabilidad y esas condiciones son, siempre y en todo caso, de orden etnocéntrico6. Para Rorty, desde esta perspectiva, las razones para juzgar conductas que pueden parecernos reprochables son etnocéntricas $y$, en modo alguno, se trata de razones que puedan imponerse universalmente de un modo no violento.

Rorty cree que:

Sería preferible enseñarles a nuestros alumnos que esas malas personas no son menos racionales, ni menos esclarecidas, ni más prejuiciosas que las buenas personas que respetamos la otredad. Más bien el problema de las malas personas es que no han tenido la misma suerte que nosotros en cuanto a las circunstancias de su crianza. (Rorty, 2000, p. 235)

\footnotetext{
${ }^{4}$ La problemática que comporta el deseo epistemológico de plantear límites disciplinarios en la cultura moderna puede considerarse con detenimiento en Popper (1972).

${ }^{5}$ La idea de despojarnos del propósito de conmensurar los discursos educativos para que estos se ajusten a una particular forma de entender la pedagogía está en sintonía con la noción de inconmensurabilidad planteada por Thomas Kuhn (1996).

${ }^{6}$ Para un análisis pormenorizado del carácter etnocéntrico de la idea de racionalidad, véase Rorty, 1996.
} 
En el marco de una educación democrática, no parece conveniente recurrir a la idea de que determinadas personas son irracionales o inmorales para explicar el tipo de conducta que consideramos reprochable. Parece más conveniente considerar a esas personas como sujetos desposeídos de seguridad y de simpatía. En este contexto, el término seguridad designa condiciones de vida lo suficientemente libres de riesgos como para convertir, en no esenciales para la propia estima y el sentido de dignidad, las diferencias con los otros. Se entiende por simpatía algo así como el tipo de reacciones que aumentaron entre los griegos después de contemplar Los Persas de Esquilo (Rorty, 2000, p. 235).

Rorty, acordando con Annette Baier, sugiere, para una educación democrática, el reemplazo de la noción de obligación por la noción de confianza, como categoría moral fundamental. El giro propuesto apunta a reflexionar sobre las ventajas pragmáticas de los fundamentos pedagógicos y la posibilidad de obtener mejores resultados educativos a partir de ese desplazamiento.

Una educación de tipo sentimental como la que sugiere Rorty no propone valores sobre la base de fundamentos sólidos y evidentes que hagan de esa propuesta una verdad universal indiscutible7. Una educación sentimental como la que propone el norteamericano no impone una jerarquía axiológica objetiva. Esos procedimientos didácticos se corresponden, no solo con una forma de entender la educación, sino también con una manera de concebir la racionalidad y la comunidad. La educación sentimental que sugiere Rorty es compatible con la idea de que nuestras decisiones morales no se fundan en un conocimiento epistemológico o de orden metaético. Por el contrario, el pragmatista duda de la eficacia de algún tipo de conocimiento de ese orden para orientar nuestra conducta moral. Los hombres no somos portadores de algún tipo de racionalidad indicativa de cómo debemos obrar.

La educación sentimental que propone el pragmatista es el tipo de educación moral y ciudadana que apela a los sentimientos, en lugar de algún tipo de cognición, para orientar la conducta de los ciudadanos. Este tipo de educación no pretende justificar racionalmente las conductas que "debemos" tener para con los demás; por el contrario, dejando de lado aquella supuesta justificación, lo que se propone es indagar en los motivos por los que "debemos" albergar ciertas conductas para con aquellas personas que nos son extrañas o para con el tipo de personas cuyas creencias son significativamente diferentes de las nuestras.

Aquel tipo de educación aspira a estimular sentimientos de solidaridad. Esta aspiración se realiza con base en las redescripciones que realizamos de los demás y de nosotros mismos. Los sentimientos de solidaridad dependen de las semejan-

\footnotetext{
7 La educación sentimental que sugiere Rorty es incompatible con una fundamentación metafísica; en lugar de ello, se articula con una disposición ironista, entendiendo por ironista "a la persona que reúna estas tres condiciones: 1) tenga dudas radicales y permanentes acerca del léxico último que utiliza [...] 2) advierta que un argumento formulado con su léxico actual no puede ni consolidar ni eliminar esas dudas; 3) [...] no piensa que su léxico se halle más cerca de la realidad que los otros" (Rorty, 1991, p. 91).
} 
zas y las diferencias con los demás que nos resulten más relevantes. Se experimentan sentimientos más profundos con quienes son, en algún sentido, más semejantes a nosotros. En cambio, podemos llegar a ser insensibles con personas muy diferentes, como con aquellos que consideramos demasiado extraños.

Las semejanzas y diferencias con los demás no constituyen el producto de un reconocimiento de verdaderas identidades ni son el fruto de un hallazgo causado por nuestra agudeza intelectual. La relevancia de las similitudes o de las diferencias para con el resto de las personas es un hecho contingente que se vincula con las descripciones que tenemos a mano, en un determinado momento histórico, de los propios hábitos y expectativas. La tarea de ampliar nuestra solidaridad solo puede realizarse minimizando las diferencias y haciendo cada vez más notorias las semejanzas que tenemos con los demás.

El progreso de nuestra solidaridad comporta:

La capacidad de percibir cada vez con mayor claridad que las diferencias tradicionales (de tribu, de religión, de raza, de costumbres y las demás de la misma especie) carecen de importancia cuando se las compara con las similitudes referentes al dolor y la humillación. (Rorty, 1991, p. 210)

Las herramientas didácticas para una educación sentimental que estimule la solidaridad en los términos señalados parecen no ser compatibles con leyes o manuales que fundamenten la obligación moral de los ciudadanos. Las reglas que impulsan esos manuales están, por lo general, atadas a fundamentos con pretensión de evidencia y universalidad; se trata de prescripciones morales impulsadas al margen de cualquier contexto social e histórico contingente. Una didáctica sentimental puede beneficiarse de relatos, cuentos o novelas que estimulen los procesos de identificación con otras personas, más allá de las diferencias que tengamos con esa gente. Las novelas de Mauro de Vasconcelos, del tipo de Barro Blanco o Mi planta de naranja lima, Oliver Twist de Charles Dickens y Las aventuras de Tom Sawyer de Mark Twain constituyen un material didáctico que puede ser de utilidad para una biblioteca de educación ciudadana. Ese tipo de herramienta didáctica no prescribe obligaciones; su función didáctica radica en sugerir y estimular formas de identificación con personajes y situaciones que propicien sentimientos nuevos.

Si el sentido de la solidaridad se basa, como quiere Rorty, en "el sentimiento de un peligro común" (Rorty, 1991, p. 107), entonces el reconocimiento de sí mismo como alguien que puede ser objeto de crueldad y humillación se vuelve relevante como motivo para desear evitar el sufrimiento de otros.

La idea de que podemos experimentar situaciones no deseadas para nosotros mismos puede llevarnos a evitar esas mismas situaciones para con otras personas. En esta perspectiva, la enseñanza de valores que apunten a evitar la crueldad o la humillación de los otros no necesita de un respaldo argumentativo que evidencie un fundamento ontológico o epistemológico. La enseñanza de esos valores puede resultar más efectiva si se sugiere trabajar la idea de un sentimiento común con los demás ciudadanos. 
Una didáctica sentimental al servicio de la democracia requiere, también, de un tipo particular de ficción. Se trata de un género literario que pueda mostrar:

El modo en que nuestros intentos de autonomía, nuestras obsesiones privadas por el logro de determinada forma de perfección, pueden hacernos olvidar el dolor y la humillación que estamos causando. Son libros en los que se dramatiza el conflicto entre los deberes para consigo mismo y los deberes para con los demás. (Rorty, 1991, p. 160)

En una comunidad democrática, los valores y las creencias de los ciudadanos merecen igual respeto. Es propio de este tipo de comunidad el estímulo de la igualdad de oportunidades para que los ciudadanos puedan desarrollar sus intereses privados sin obstaculizar las oportunidades de sus conciudadanos.

El sentido de una comunidad democrática radica en ofrecer a todos sus ciudadanos la libertad y la igualdad para la realización de sus proyectos privados. En este sentido, una educación de carácter democrático es aquella que admite, sin extrañeza, la pluralidad de diferencias artísticas, literarias, religiosas y hasta políticas. La educación conveniente para este tipo de comunidad no pretende uniformar la cultura o las opiniones de sus ciudadanos. Por el contrario, la mejor educación para una comunidad democrática es aquella que pueda ofrecer una pluralidad de perspectivas al interior de cada geografía cultural sin que ninguna de ellas tenga primacía por sobre las demás. En este sentido, la educación ciudadana, que en este contexto no puede ser sino democrática, puede hacerse efectiva desde cualquier parcela del currículo escolar. De lo que se trata es de ofrecer la posibilidad de redescribir las perspectivas ajenas sin considerarlas insignificantes o menos importantes que la propia, por el solo hecho de no ser la perspectiva propia. Los intereses estéticos, literarios o filosóficos de los demás no son menos valiosos que el propio por ser ajeno; simplemente no son propios. Sin embargo, por eso mismo, pueden ser una ocasión para redescribir las propias creencias y expectativas.

En resumidas cuentas y a modo de conclusión, podemos afirmar que "pedagogía" y "posmodernidad" no constituyen expresiones excluyentes o, al menos, no son excluyentes si la noción de pedagogía no es constitutivamente moderna. Si no concebimos la pedagogía como una parcela cultural necesariamente amarrada a una epistemología con pretensiones de conmensuración, nada impedirá imaginar las posibilidades de una pedagogía posmoderna.

La propuesta rortyana de una educación sentimental constituye una herramienta pedagógica que puede ser de utilidad para una educación democrática. Esbozar un diseño de procedimientos didácticos sobre el desplazamiento de la noción de obligación moral en dirección a la noción de confianza puede ser una ocasión para reflexionar sobre las propias prácticas educativas y abrir la puerta a la obtención de resultados deseados para la escolaridad actual. Si acaso esos resultados no se condicen con nuestras expectativas, no habremos perdido mucho, si consideramos que las prácticas educativas actuales no parecen acertar con los objetivos de nuestros sistemas educativos. 


\section{Una pedagogía entre la epistemología y la hermenéutica}

Para el filósofo norteamericano, el término "filosofía" es un término ambiguo que puede ser utilizado con significaciones muy diversas. No existe algo semejante a "la filosofía", puesto que la filosofía no comporta ninguna esencia. En la historia de la filosofía, pueden distinguirse dos tipos muy diferentes de filósofos: Ios pensadores cuyas obras son esencialmente constructivas y aquellos intelectuales cuyas obras son esencialmente reactivas (Rorty, 1995, p. 331). El contraste entre los filósofos constructivos y los filósofos reactivos es el contraste entre aquellos que se centran en la epistemología y quienes, por el contrario, mantienen una actitud de sospecha y desconfianza hacia las pretensiones epistemológicas de la filosofía.

Los filósofos, constructivos o sistemáticos, se caracterizan por la pretensión de poseer un conocimiento verdadero y evidente. Pero, además de los filósofos sistemáticos, existen también figuras filosóficas periféricas o reactivas; estas son figuras intelectuales que "se emparentan entre sí por su desconfianza para con la idea de que la esencia del hombre es la de un ser conocedor de esencias" (Rorty, 1995 , p 332). Estos pensadores entienden que nuestras creencias, vocabularios y lenguajes no expresan representaciones privilegiadas respecto de la esencia de las cosas, sino que constituyen creencias y vocabularios particulares en el concierto de infinitas creencias e infinitos vocabularios posibles. Para estos filósofos periféricos, nuestro vocabulario se asemeja a una herramienta más, entre otras herramientas posibles, a la mano de las personas para el logro de sus propósitos. A estos pensadores periféricos, respecto de las corrientes dominantes de la historia de la filosofía de occidente, Rorty los denomina filósofos edificantes.

El contraste entre los filósofos sistemáticos y los filósofos periféricos evidencia dos modos alternativos de concebir la verdad y, por ende, de comprender la filosofía.

Echando mano al lenguaje de Thomas Kuhn, Rorty ofrece otra distinción: aquella que distingue a los filósofos normales de los filósofos revolucionarios. El filósofo normal no tiene dudas respecto de los problemas que discute; su actitud es la de alguien que reconoce que sus discusiones giran en torno a problemas reales y no necesita cuestionar los términos en que esos problemas se discuten (Rorty, 2000, p. 300). Filósofo revolucionario, en cambio, es aquel que sostiene que todo, o la mayor parte del trabajo realizado por sus antecesores en un área determinada, se ha basado en un error (Rorty, 2000, p. 300). La distinción entre sistemáticos y edificantes no es equivalente a la diferencia entre filósofos normales y filósofos revolucionarios (Rorty, 1995, p. 333) ya que se pueden distinguir dos tipos de actitudes revolucionarias: la de aquellos filósofos que, a partir de una ruptura ontológica y/o epistemológica, aspiran a fundar nuevas escuelas dentro de las cuales practicar una nueva "normalidad filosófica" y la actitud de los revolucionarios edificantes (Rorty, 1995, p. 333). Para un revolucionario sistemático, la inconmensurabilidad de su vocabulario para con los vocabularios antiguos es un problema pasajero. La institucionalización del nuevo léxico propuesto y el uso, por parte de sus predecesores, de este nuevo vocabulario resuelve las diferencias. El revolucionario edificante, a diferencia del revolucionario sistemático, no tiene la pretensión de que su nuevo vocabulario sea conmensurable con el de la tradición. 
"La idea de un filósofo edificante constituye una paradoja" (Rorty, 1995, p. 333). La figura de un filósofo edificante comporta la dificultad de conciliar filosofía y edificación. La consideración de este tipo de intelectual como filósofo es posible en la medida en que el pensador utilice el lenguaje institucionalizado por la comunidad filosófica. Sin embargo, su carácter de edificante reside justamente en la utilización de un vocabulario que no sea de este tipo.

La paradoja del filósofo edificante no reside en la utilización de un vocabulario revolucionario (lo que también hace el filósofo revolucionario sistemático), sino en la utilización de un vocabulario que, además de ser revolucionario, no es considerado filosófico (Rorty, 1995, p. 334). La revolución que promueve el filósofo edificante tiene lugar en un metanivel respecto de las revoluciones que impulsan los filósofos sistemáticos. Los filósofos edificantes no sugieren un mero cambio de reglas que encajan mejor que las reglas institucionalizadas para dar cuenta de la realidad, como lo hacen los filósofos sistemáticos. Los filósofos edificantes desean que olvidemos la idea de que la filosofía debe proceder según algún tipo de regla (Rorty, 1995).

El desmontaje terapéutico que Rorty realiza de la epistemología en La filosofía y el espejo de la naturaleza puede contribuir en la discusión que impulsan Ayuste González y Trilla Bernet (2005). Las exigencias de los catalanes para que un discurso educativo constituya una pedagogía revela una actitud que puede emparentarse con la idea de una "pedagogía sistemática", esto es, una pedagogía que procede con arreglo epistemológico y el propósito de delimitar qué es y cómo "debe ser" una auténtica educación.

Los discursos educativos que no reúnen las exigencias epistemológicas de Ayuste González y Trilla Bernet comportan léxicos alternativos e inconmensurables y, por esa misma inconmensurabilidad, no gozan del privilegiado estatus epistemológico que los ubicaría en el ágora de una clasificación pedagógica. Se trata de discursos que pueden ser considerados como "periféricos y reactivos", en el lenguaje de Rorty. Esos discursos enfrentan la paradoja de una "pedagogía edificante"8 y no pretenderían lograr una representación ajustada de la esencia de la educación.

La pedagogía, entendida como la reflexión sobre las condiciones de posibilidad de representaciones y vocabularios ajustados al auténtico modo de ser de la educación, constituye la actitud propia y esencial de un "pedagogo sistemático". Una "pedagogía edificante" procedería, en cambio, y en términos de Rorty, de manera hermenéutica. La hermenéutica, como la entiende el norteamericano, no debe ser considerada como una sucesora de la epistemología. Una pedagogía que proceda de manera hermenéutica no aspira a constituir un discurso que pretenda reflejar el verdadero sentido de la educación o de evidencias pedagógicas ajustadas a una naturaleza humana. Una pedagogía de corte hermenéutico no

\footnotetext{
${ }^{8}$ La idea de una "pedagogía edificante" aparenta una redundancia si se entiende que el término "edificante" que usa Rorty comporta la recuperación del sentido de la expresión que "Bildung" tiene en la obra de Gadamer. No obstante, la expresión alude a un carácter que puede ser atribuido tanto a la pedagogía como a otras disciplinas. En el caso de Rorty, la expresión hace referencia al carácter propio de un modo de concebir la filosofía. Para una consideración de la expresión en la obra de Gadamer, véase Gadamer, 2015.
} 
pretende ocupar el lugar, en desuso, de las pedagogías de la modernidad. Hermenéutica constituye solo otro nombre para el ocaso de la epistemología. Los denominados "discursos educativos", que no comportan el estatus epistemológico de las pretendidas "auténticas pedagogías", pueden contribuir a fortalecer las discusiones educativas actuales.

Quizás desde las periferias de la pedagogía sistemática puedan emerger discusiones innovadoras, propuestas como la de una educación sentimental y nuevos léxicos para estimular conversaciones aún no imaginadas sobre nuestras propias prácticas educativas.

\section{Referencias bibliográficas}

Ayuste González, A. \& Trilla Bernet, J. (2005). Pedagogías de la modernidad y discursos postmodernos sobre la educación. Revista de educación, 336, 219-248.

Gadamer, H. G. (2015). Verdad y método I. Salamanca: Sígueme.

Giroux, H. (1993). La escuela y la lucha por la ciudadanía. México: Siglo XXI.

Kuhn, T. (1996). La estructura de las revoluciones científicas. Buenos Aires: Fondo de cultura económica.

Laudo Castillo, X. (2011). La hipótesis de la pedagogía moderna. Educación, verdad y relativismo. Teoría educativa, 43, 45-68.

Popper, K. (1972). Conjeturas y refutaciones. El desarrollo del conocimiento científico. Barcelona: Paidós.

Rorty, R. (1991). Contingencia, ironía y solidaridad. Barcelona: Paidós.

Rorty, R. (1993). Ensayo sobre Heidegger y otros pensadores contemporáneos. Escritos filosóficos 2. Barcelona: Paidós.

Rorty, R. (1995). La filosofía y el espejo de la naturaleza. Madrid: Cátedra.

Rorty, R. (1996). Objetividad, relativismo y verdad. Escritos filosóficos I. Barcelona: Paidós.

Rorty, R. (1998a). Pragmatismo y política. Barcelona: Paidós.

Rorty, R. (1998b). Respuesta a Ernesto Laclau. En S. Critchley, J. Derrida, E. Laclau, R. Rorty \& Ch. Mouffe, Deconstrucción y pragmatismo (pp. 135-146). Buenos Aires: Paidós.

Rorty, R. (2000). Verdad y progreso. Escritos filosóficos 3. Barcelona: Paidós.

Rorty, R. (2005). Cuidar la libertad. Entrevistas sobre política y filosofía. Edición de Eduardo Mendieta. Madrid: Trotta. 